\title{
ARTICLE \\ T-bet optimizes CD4 T-cell responses against influenza through CXCR3-dependent lung trafficking but not functional programming
}

\author{
Kunal Dhume ${ }^{1}$, Caroline M. Finn ${ }^{1}$, Tara M. Strutt ${ }^{1,2}$, Stewart Sell ${ }^{3}$ and K. Kai McKinstry ${ }^{1,2}$
}

\begin{abstract}
Although clearance of many intracellular pathogens requires T-bet-dependent CD4 T cell programming, the extent to which T-bet is needed to direct protective CD4 responses against influenza is not known. Here, we characterize wild-type and T-bet-deficient CD4 cells during murine influenza infection. Surprisingly, although T-bet expression has broad impacts on cytokine production by virusspecific CD4 cells, the protective efficacy of T-bet-deficient effector cells is only marginally reduced. This reduction is due to lower CXCR3 expression, leading to suboptimal accumulation of activated T-bet-deficient cells in the infected lung. However, T-betdeficient cells outcompete wild-type cells to form lung-resident and circulating memory populations following viral clearance, and primed T-bet-deficient mice efficiently clear supralethal heterosubtypic influenza challenges even when depleted of CD8 T cells. These results are relevant to the identification of more incisive correlates of protective $T$ cells and for vaccines that aim to induce durable cellular immunity against influenza.
\end{abstract}

Mucosal Immunology (2019) 12:1220-1230; https://doi.org/10.1038/s41385-019-0183-z

\section{INTRODUCTION}

CD4 T cells combat pathogens through direct effector functions and by helping to maximize the protective activities of other leukocytes. ${ }^{1}$ There is increasing interest in improving the ability of vaccines to prime CD4 immunity against threats like Influenza $A$ virus (IAV) that can escape antibody-mediated protection. Prerequisite for such approaches is establishing the kinds of CD4 responses needed to clear a given microbe. This question has been framed for the last 30 years by the expanded 'Th1/Th2' paradigm that categorizes CD4 cells largely based on their cytokine production. In general, protection against intracellular pathogens is believed to require Th1-polarized cells characterized by strong IFN $\gamma$ production and a broader differentiation program guided by the 'master' transcription factor T-bet. ${ }^{2}$

A number of functionally distinct subsets of CD4 T cells combat IAV using multiple mechanisms that provide synergizing and redundant layers of protection. ${ }^{3,4}$ A complete description of the distinct mechanisms brought to bear as part of this integrated response is still evolving, but an implicit assumption is that T-betdependent programming is crucial to successful CD4 T cellmediated IAV clearance. Seminal work found that Th1-polarized clones recognizing IAV could transfer immunity to unprimed hosts while Th2 clones could not. ${ }^{5}$ Subsequent studies showed that IAVspecific Th1 effector or memory cells also protect naïve mice while Th2 and unpolarized (Th0) cells do not., ${ }^{3,6}$ Furthermore, IFNy production is the hallmark of CD4 cells responding to IAV and in some models CD4 T cell protection is IFNY-dependent. ${ }^{7-9}$ Indeed, IFNy remains by far the most measured CD4 attribute across human and animal IAV studies, supporting the consensus that Th1 responses underlie effective CD4 T cell immunity.
Some evidence, however, indicates that prototypical Th1 cells may not be needed for robust immunity against IAV. For example, IFNY-deficient mice have been shown to be no more susceptible to IAV than WT mice, ${ }^{10}$ and we found IFNY neutralization not to compromise the ability of Th1-polarized memory cells to protect naïve WT mice. ${ }^{3}$ In fact, ablating IFNy signaling can reduce morbidity during IAV infection, correlating with improved innate lymphoid cell function ${ }^{11}$ and reduced viral spread. ${ }^{12}$ Additionally, IAV-specific Th17 cells can protect naïve mice against $I A V^{13}$ and may contribute to vaccine-primed immunity. ${ }^{14}$

To determine how T-bet expression affects the overall development of protective CD4 effector and memory responses we analyzed WT and T-bet-deficient $\left(T b \times 21^{-/-}\right)$T cell receptor transgenic as well as polyclonal CD4 cells responding against IAV. Whereas T-bet does not impact activation, it has a broad impact on effector cytokine production, highlighted by decreased IFN $\gamma$ and increased IL-17 production by $T b \times 21^{-/-}$vs. WT CD4 $\mathrm{T}$ cells. CD4 $\mathrm{T}$ cell-intrinsic T-bet is also required for maximal effector accumulation in the lung. Higher expression of the known T-bet-regulated chemokine receptor CXCR3 ${ }^{15}$ is alone responsible for increased WT vs. Tbx $21^{-1-}$ CD4 responses in the lung. We transferred effector cells primed in vitro under "Th1" conditions to naive mice and challenged with IAV to determine the extent that T-bet impacts their anti-viral capacity. While WT and Tbx $21^{-1-}$ effectors both protect against lethal IAV, ultimately the input of fewer WT donors is required, reflecting the marginally compromised lung trafficking of $T b \times 21^{-1-}$ effectors.

Tbx $21^{-1-}$ effectors at the peak of the anti-viral response display hallmarks of a phenotype more associated with memory precursor cells than do WT effectors and they outcompete WT CD4 cells to

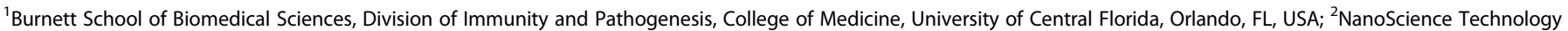
Center, University of Central Florida, Orlando, FL, USA and ${ }^{3}$ Wadsworth Center, Albany, NY, USA

Correspondence: K Kai McKinstry (kai.mckinstry@ucf.edu)

Received: 10 January 2019 Revised: 9 May 2019 Accepted: 4 June 2019

Published online: 5 July 2019 

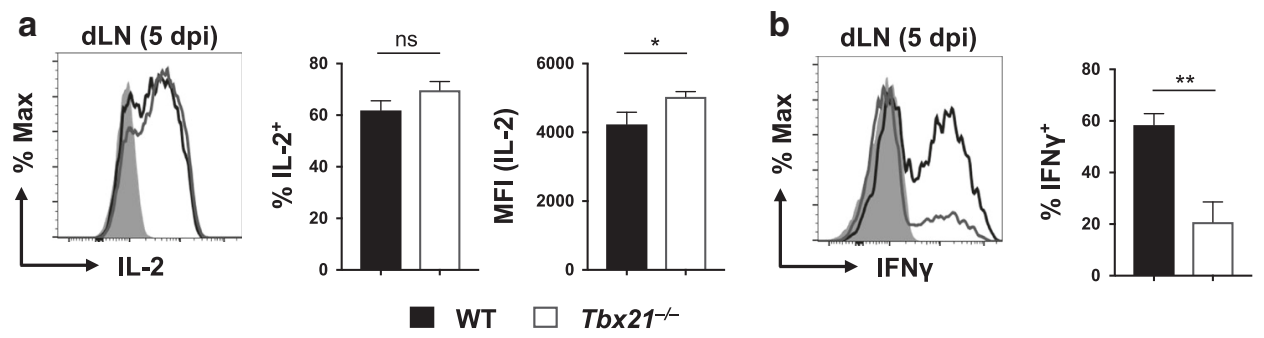

C

dLN (5 dpi)
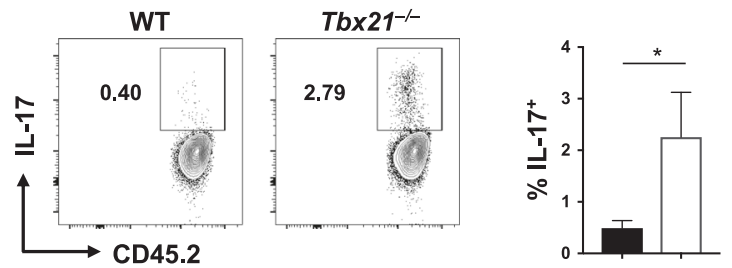

d

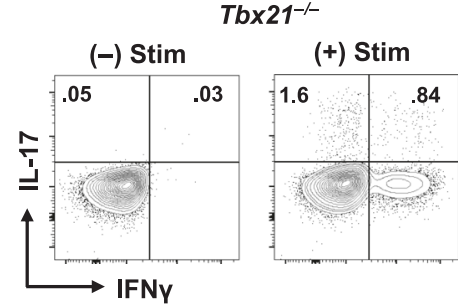

Fig. 1 Increased IL-17 and diminished IFNY production from early Tbx $21^{-1-}$ effectors primed by IAV. $1 \times 10^{6}$ naïve WT or Tbx21-/- OT-II cells were adoptively transferred to congenic (CD45.1 $1^{+}$) WT hosts and challenged with a sublethal dose of PR8-OVA A. $_{\text {. At }} 5 \mathrm{dpi}$, dLNs were harvested and cytokine production assessed from donor cells by intracellular staining. a Representative staining for IL-2 from WT (black) and Tbx21 ${ }^{-1-}$ (grey) cells and control staining of WT cells without re-stimulation (solid) along with a summary of the percent of IL-2 positive cells (left) and MFI (right) for each population ( $n=4$ mice/group). b IFN $\gamma$ production with representative staining (left) and the percent of positive cells (right). c Representative staining for IL-17 from WT (left) and Tbx $21^{-1-}$ (right) cells with summary from 4 mice/group, and (d) representative staining of IL-17 vs. IFN $\gamma$ from $\mathrm{Tb} \times 21^{-/-}$cells. Results presented from 1 of 2 similar experiments

form lung-resident and circulating memory cells after viral clearance. Finally, we find IAV-primed WT and $T b \times 21^{-/-}$mice are similarly protected against supralethal heterosubtypic IAV challenge, even when the primed mice are depleted of CD8 cells prior to secondary challenge. Our results indicate that the establishment of protective CD4 cell memory and robust secondary CD4 T cell responses against IAV do not require CD4 cell-intrinsic or extrinsic T-bet expression.

In summary, we find that classic Th1 programming is not needed for CD4 effectors to promote efficient IAV clearance. However, two important findings emerge with relevance to vaccination. First, T-bet-dependent CXCR3 levels maximize CD4 effector accumulation in the lung. In fact, we suggest CXCR3 expression is the most insightful T-bet-dependent correlate of protective CD4 cells. Second, T-bet reduces the memory fitness of IAV-primed CD4 cells. This indicates that T-bet levels induced during priming must be carefully balanced in order to promote effector cells programmed with maximal anti-viral capacity without sacrificing their ability to form optimal long-lived memory.

\section{RESULTS}

T-bet-deficiency prevents development of Th1 but not Th2 or Th17 cells in vitro

To determine the role of T-bet in CD4 responses against IAV, we bred $T b \times 21^{-/-}$OT-II TcR transgenic mice recognizing an epitope of ovalbumin (OVA) expressed by the A/PR8-OVA $A_{\|}$virus. Prior to experiments with IAV, we confirmed the phenotype of $T b \times 21^{-/-}$ effector cells generated in defined priming environments in vitro using antigen presenting cells and $\mathrm{OVA}_{\|}$peptide. As expected, Tbx $21^{-1-}$ cells primed in "Th1" conditions did not show the strong IFN $y$ production seen from WT cells (Supplementary Fig. 1). Th2 cytokines were not produced by $T b \times 21^{-1-}$ "Th1" effectors (not shown), nor was IL-17 (Supplementary Fig. 1). T-bet-deficiency did not alter expression of key transcription factors or cytokines in Th2 or Th17 cells, or in unpolarized Th0 cells (Supplementary Fig. 1). Finally, T-bet did not impact expansion in any condition (Supplementary Fig. 1). These results confirm a need for T-bet to program prototypical 'Th1' responses marked by strong IFN $\gamma$ production.
T-bet promotes maximal lung CD4 responses and broadly impacts cytokine production

We transferred $1 \times 10^{6}$ naïve CFSE-labeled WT or Tbx $21^{-1-}$ OT-II cells to $\mathrm{CD} 45.1^{+} \mathrm{B} 6$ hosts and challenged with a sublethal $(0.2$

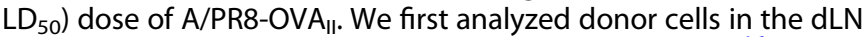
at $5 \mathrm{dpi}$, before their appreciable migration to the lung. ${ }^{16}$ Similar numbers of WT and $T b \times 21^{-1-}$ donor cells were present, and all were CFSE ${ }^{\text {low }}$ (not shown). WT and Tbx $21^{-1-}$ cells displayed similar IL-2 production, but more IL-2 $2^{\text {high }}$ cells were seen in $\mathrm{Tb} \times 21^{-/-}$cells, resulting in increased MFI at the population level (Fig. 1a). Consistent with results in vitro, Tbx $21^{-1-}$ cells made less IFNY (Fig. 1b) but a small percentage also produced IL-17 while almost no $\mathrm{IL}^{-17^{+}}$WT cells were seen (Fig. 1c). Both $\mathrm{IFNy}^{+}$and $\mathrm{IFN \gamma}^{-}$ Tbx $21^{-1-}$ cells were $\mathrm{IL}-17^{+}$(Fig. $1 \mathrm{~d}$ ), indicating diverse cytokine production patterns within the $T b \times 21^{-/-}$effectors. Thus, T-bet does not impact the kinetics of CD4 T cell activation but does alter the functional potential of early effector cells primed by IAV infection.

The frequency and number of WT and Tbx $21^{-/-}$cells at $7 \mathrm{dpi}$ (the peak of donor CD4 responses) were similar in the spleen and dLN, but $T b \times 21^{-1-}$ cells in the lung reached only about one half of the WT response (Figs. 2a, b). Lower Tbx $21^{-/-}$counts were not due to less proliferation as Ki67 staining in WT and Tbx21 $1^{-1-}$ cells was comparable (Fig. 2c). Decreased $T b \times 21^{-1-}$ cell accumulation correlated with their slightly lower expression of the integrin CD11a (Fig. 2d) and the chemokine receptor CXCR3 (Fig. 2e). CD11a supports CD4 lung trafficking during mycobacterial challenge, ${ }^{17}$ and CXCR3, a known T-bet target, ${ }^{15}$ optimizes lung CD4 trafficking in a parainfluenza model. ${ }^{18}$ In contrast, $T b \times 21^{-/-}$ cells expressed slightly more CCR4 (Fig. 2f), which is also linked to CD4 lung trafficking during IAV infection, ${ }^{19}$ but both cell types expressed many other chemokine receptors similarly including CXCR5, CCR5, CCR6, and CCR7 (not shown). Thus, T-bet expression by CD4 cells is required for maximal effector responses in the lung, possibly through regulating cell trafficking.

Analysis of cytokine production at $7 \mathrm{dpi}$ by effectors responding in the lung revealed reduced IFN $y$ and increased IL-2 by $T b \times 21^{-/-}$ cells (Fig. 3a), a pattern also seen in the spleen and dLN (Supplementary Fig. 2). A sizable cohort of $T b \times 21^{-/-}$but not WT effectors produced IL-17 (Fig. 3a), while little IL-17 was seen 
a
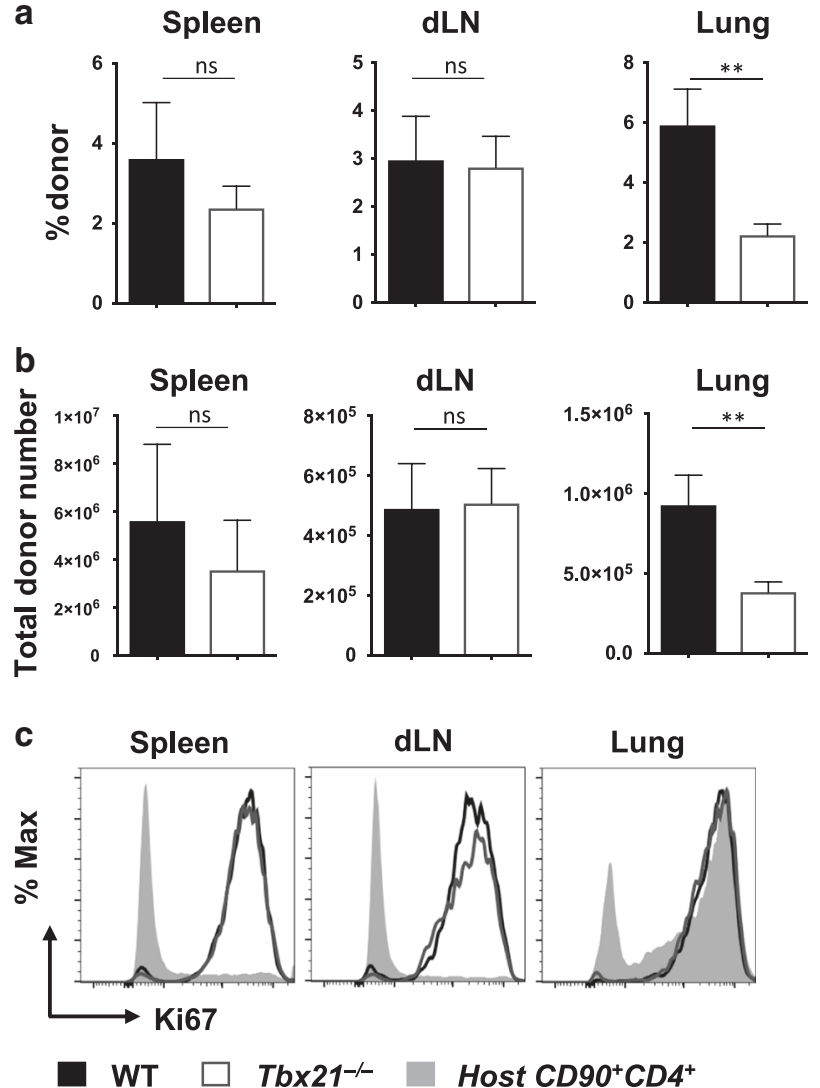

dLN

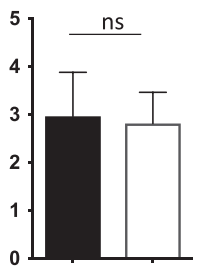

dLN

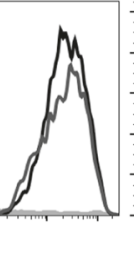

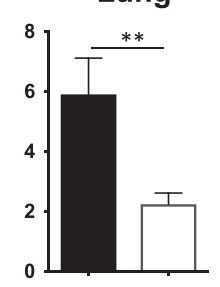
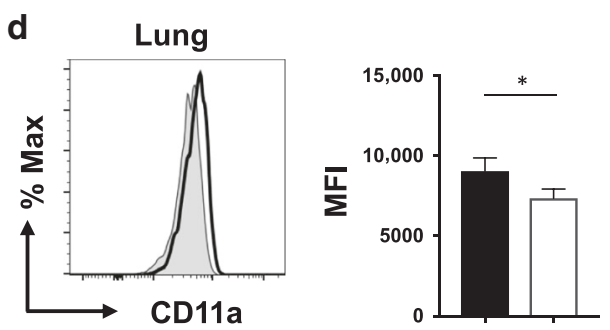

e
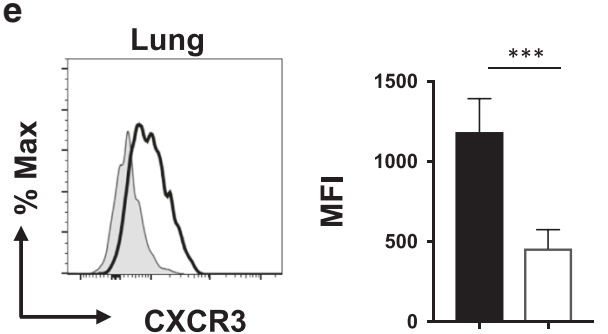

f
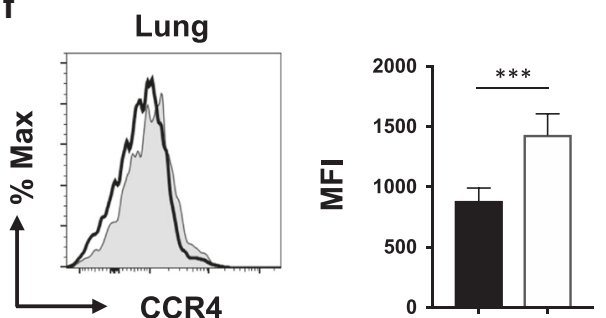

Fig. 2 Reduced lung accumulation and differential expression of homing markers by Tbx $21^{-/-}$effector cells. $1 \times 10^{6}$ naïve WT or Tbx2 $1^{-/-}$OT-II cells

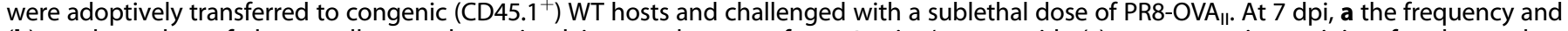
(b) total number of donor cells was determined in stated organs from 4 mice/group, with (c) representative staining for the nuclear proliferation marker Ki67 shown in stated organs for host CD4 T cells (solid), WT OT-Il cells (black) and Tbx21 d Representative staining (left) and accompanying MFI analysis (right) from donor cells in the lungs from 3 mice/group for (d) CD11a, (e) CXCR3, and (f) CCR4. Results presented from 1 of 3 similar experiments

outside of the lung (Supplementary Fig. 2). There was no difference in IL-10 production, which was limited to the lungs as established previously ${ }^{13}$ (Fig. 3a). TNF levels were not impacted by T-bet (Fig. 3a and Supplementary Fig. 2), but increased IL-4 and reduced GM-CSF were seen from $\mathrm{Tb} \times 21^{-1-}$ cells in all organs tested (Fig. 3a and Supplementary Fig. 2). T-bet expression thus impacts diverse aspects of cytokine production by IAV-primed CD4 T cells, especially in the lung (Fig. 3b). WT and Tbx $21^{-1-}$ cells expressed similar levels of Granzyme B, with higher levels in lung than spleen (Fig. 3c), a pattern consistent with previous studies. ${ }^{6}$

Virtually all WT effector cells in the lung expressed T-bet at 7 dpi (Fig. 3d) that, together with high IFNY and low IL-4 and IL-17 production, clearly mark the WT response against IAV as strongly Th1-polarized. Increased IL-17 and IL-4 production by Tbx $21^{-1-}$ cells correlated with $\sim 30 \%$ staining for Roryt and $\sim 4 \%$ for GATA-3 (Fig. 3d). These results indicate CD4 T cell-intrinsic Tbet represses Th17 and Th2 elements that can otherwise be directed by infection-induced signals. Very few $\mathrm{FoxP}^{+}$donor cells were detected, indicating that $T b \times 21^{-/-}$cells are not more prone to Treg differentiation than are WT cells (Fig. 3d). Finally, we observed similar frequencies of follicular helper cells $\left(T_{F H}\right)$ within WT and $\mathrm{Tb} \times \mathrm{1}^{-1-}$ effectors in the spleen and dLN (Fig. 3e).

It is critical to confirm key results made with transgenic OT-II cells with endogenous polyclonal CD4 T cell responses. Because Tbet is expressed by adaptive and innate immune cells, ${ }^{20}$ and could thus indirectly impact CD4 T cell responses in Tbx21-1- mice, we created chimeras using 50/50\% WT (Thy1.1/Thy1.2) and Tbx21 1 -1(Thy1.2) bone marrow to reconstitute lethally irradiated Thy1.1 B6 hosts. This approach allows WT and $T b \times 21^{-1-}$ cells responding in the same environment to be clearly resolved (Fig. 4a). After validating chimera establishment, we challenged with $A / P R 8$, and IAV-specific CD4 cells were visualized using a tetramer for the major $1-A^{b}$ restricted epitope $\mathrm{NP}_{311-325}$ (Fig. 4b). Similar frequencies of WT and $\mathrm{Tb} \times 21^{-1-} \mathrm{NP}_{311}{ }^{+}$cells were seen in the spleen and dLN at $9 \mathrm{dpi}$, but $>70 \%$ of tetramer ${ }^{+}$cells in the lungs were WT (Fig. 4c). Combined with lower CXCR3 expression by polyclonal Tbx $21^{-1-}$ cells (Fig. 4d), these findings are consistent with results from OT-II cells above. The lung CD4 ${ }^{+} \mathrm{CD} 44^{\text {high }} \mathrm{Tb} \times 21^{-/-}$cells were also marked by reduced IFNY and increased IL-17 and IL-2 (Figs. $4 \mathrm{e}, \mathrm{f}$ ), again confirming hallmarks of the WT vs. Tbx $21^{-/-}$OTII response.

CXCR3-mediated signals alone lead to improved lung responses from WT CD4 cells

We next addressed the extent to which CXCR3-mediated signals account for greater WT vs. Tbx $21^{-1-}$ CD4 T cell accumulation in infected lungs. We co-transferred equal numbers of WT (Thy1.1/ Thy 1.2) and Tbx21-1- (Thy1.2) OT-II cells to CD45.1 ${ }^{+}$B6 mice, challenged with PR8-OVA $A_{l 1}$, and treated one group of recipients with blocking antibody against CXCR3 from 3-6 dpi and the other with PBS (Fig. 5a). We did not block CXCR3 directly after infection as CXCR3-mediated interactions can impact CD4 T cell activation. ${ }^{21}$ At 7 dpi we confirmed CXCR3 blockade (Figs. 5b, c) and assessed donor cell accumulation in the lungs. While Tbx $21^{-1-}$ responses were largely unaffected by CXCR3 blockade, WT cells were reduced by about half (Fig. $5 \mathrm{~d}$ ), leading to similar numbers of WT and $T \times b 21^{-/-}$cells. We asked if a particular 

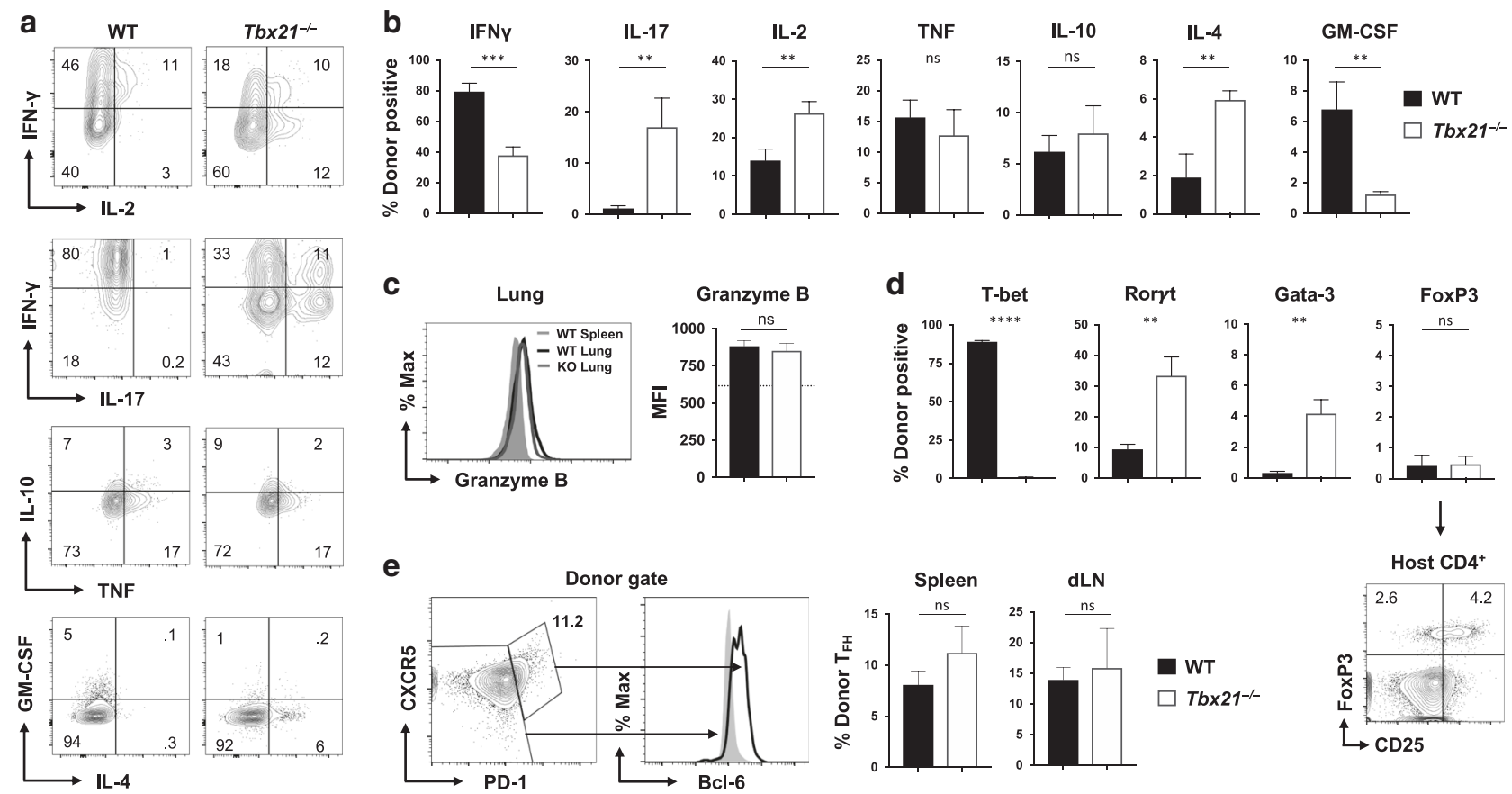

Fig. 3 T-bet controls diverse aspects of cytokine production by effector cells primed by IAV. a Representative staining for stated combinations of cytokines are shown for donor cells in the lung at $7 \mathrm{dpi}$ with (b) summary analysis for individual cytokine production from 4 mice/group. c Representative staining for granzyme B is shown for WT (black) and Tbx $21^{-1-}$ cells (grey) in the lungs vs. WT cells in the spleen (solid), with MFI analysis from 3 mice per group (dotted line represents average spleen MFI for granzyme B). Results representative of 3 similar experiments. d Donor effectors in the lung were also analyzed for expression of transcription factors T-bet, Roryt, GATA3, and FoxP3 at 7 dpi, with the frequency of positive donors cells shown from 4 mice/group. Representative staining of host CD4 T cells for FoxP3 and CD25 is also shown as a staining control. One of 3 separate experiments. e Follicular helper cells $\left(T_{\mathrm{FH}}\right)$ within WT and Tbx21 $1^{-1-}$ donor cells at 7 dpi was determined as depicted using CXCR5, PD1, and Bcl-6, with $\mathrm{T}_{\mathrm{FH}}$ frequency shown for the spleen (left panel) and dLN (right panel) from 4 mice per group. 1 of 2 separate experiments

subset of WT effectors was impacted by CXCR3 blockade, but extensive phenotyping (not shown), and analysis of cytokine production (Fig. 5e) revealed similar cohorts of WT cells in the lung with or without CXCR3 blockade. T-bet-dependent CXCR3mediated signaling is thus entirely responsible for improved accumulation of WT vs. T-bet-deficient CD4 effectors in IAVinfected lungs.

Tbx $21^{-1-}$ CD4 effectors are protective against lethal IAV infection For decades, effective CD4 T cell responses against IAV have been characterized as strongly Th1-polarized. This conclusion rests largely on using differentially-polarized effector cells to transfer immunity to unprimed mice. We used this approach to compare the protective efficacy of an equal number of WT or Tbx $21^{-1-}$ OT-II effectors generated in 'Th1' conditions (as in Supplementary Fig. 1). We first transferred $3 \times 10^{6}$ cells and found that both effectors protected against lethal PR8-OVA that recipients of $T b \times 21^{-1-}$ cells lost around $10 \%$ more weight (Fig. 6a). Nevertheless, recipients of both effector types similarly gained weight back and showed no difference in viral clearance vs. control mice not receiving donor cells that succumbed to infection (Fig. 6b). T-bet programing is thus largely dispensable for CD4 effector-mediated protection against even lethal IAV challenge.

The responding $T b \times 21^{-1-}$ and WT effectors displayed similar differences in cytokine production as effectors arising from naïve CD4 cells shown in Fig. 3, highlighted by reduced IFNY and GM-CSF, and enhanced IL-2, IL-4, and IL-17 (not shown). Given that increased IL-17 production is the most remarkable functional distinction between WT and $T b \times 21^{-1-}$ cells, and that $\mathrm{IL}-17$ may either be protective during IAV infection ${ }^{22}$ or drive immunopathology and weight loss, ${ }^{23}$ we treated mice receiving Tbx $21^{-1-}$ effectors with antibody to neutralize IL-17 or an isotype control. Blocking IL-17 caused only a minor reduction in weight loss (Fig. 6c) and had no impact on viral control (Fig. 6d). IL-17 is thus neither a critical component of the protective Tbx $21^{-/-}$CD4 response, nor a major driver of weight loss during IAV challenge.

CXCR3 is required to maximize the efficacy of CD4 T cell effectormediated protection

We next assessed the number of WT or $T b \times 21^{-1-}$ donor effectors at $5 \mathrm{dpi}$, the peak of their response in this model. No differences were seen in the spleen or $\mathrm{dLN}$, but fewer $\mathrm{Tb} \times 21^{-/-}$effectors were seen in lungs (Fig. 6e), similar to findings using naive cell transfers. We hypothesized that titration of the effectors transferred should thus reveal a defect in $T b \times 21^{-/-}$effector-mediated protection, as their number in the lung could drop below a required threshold earlier than for WT effectors. Indeed, when $1 \times 10^{6}$ effector cells were transferred, WT recipients began to recover by 9 dpi while mice receiving $\mathrm{Tb} \times 1^{-1-}$ cells did not (Fig. $6 \mathrm{f}$ ) and had higher viral titers (Fig. 6g).

Finally, we asked if we could recapitulate the slightly impaired protection provided by $T b \times 21^{-1-}$ effectors by blocking CXCR3 in recipients of $3 \times 10^{6}$ WT effectors. Indeed, anti-CXCR3 treatment from 1-7 dpi significantly increased weight loss and delayed recovery of adoptive hosts that were nevertheless still protected (Fig. 6h). These results indicate that T-bet-dependent CXCR3 expression by CD4 effectors is needed for their optimal protective potential, but that T-bet-dependent cytokine production is not needed for them to mediate efficient anti-viral responses. 
a

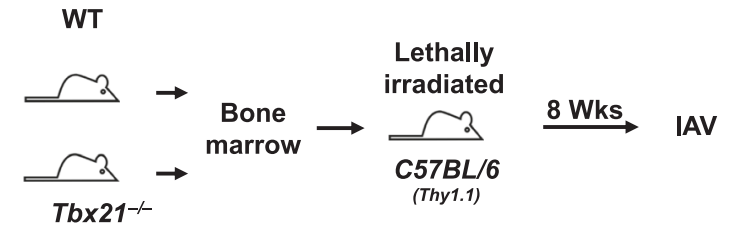

b

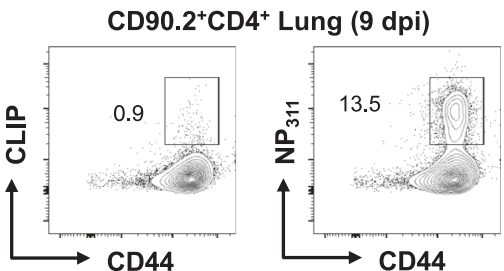

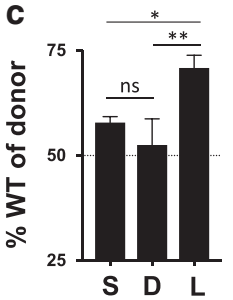
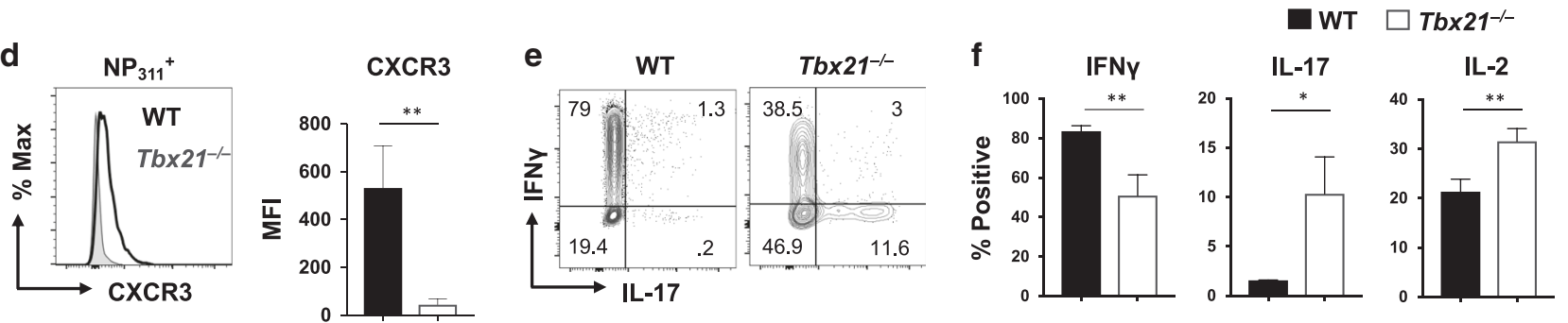

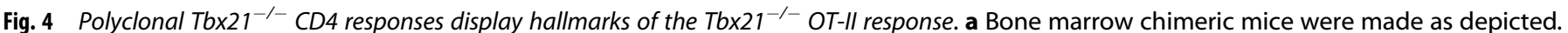
b Following sublethal PR8 infection, IAV-specific cells were detected at 9 dpi using the $\mathrm{NP}_{311}$ tetramer compared to staining with CLIP control tetramer as shown in representative panels. c The average frequency of WT vs. Tbx $21^{-/}$within the polyclonal NP $\mathrm{N}_{311}{ }^{+}$population is shown for 3 mice/group for spleen (S) dLN (D), and lungs (L). d Representative staining of CXCR3 from WT and Tbx21 $1^{-1-}$ NP311 $1^{+}$cells in the lung (left) and MFI analysis from 3 mice/group (right), and (e) representative intracellular staining for IFN $\gamma$ vs. IL-17 from WT (left panel) vs. Tbx21 ${ }^{-1-}$ (right panel) $\mathrm{CD} 44^{\text {high }} \mathrm{CD} 4$ cells in the lung at 9 dpi (e). f Summary from 3 mice/group is shown for the stated cytokines detected from CD44 ${ }^{\text {high }}$ CD4 cells in the lung. Results from one of two similar experiments

a

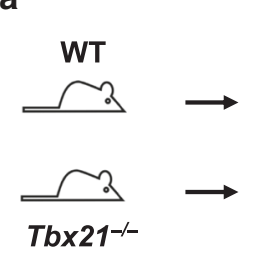

C

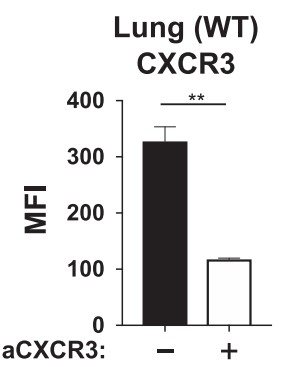

Naïve OT-II cells

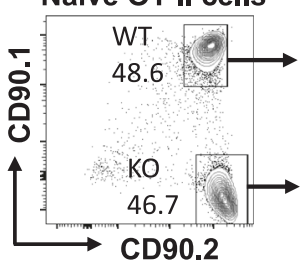

d

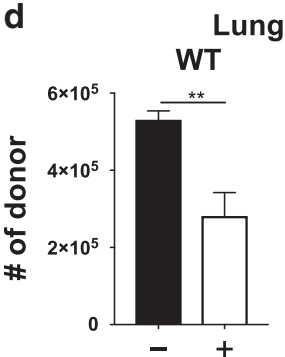

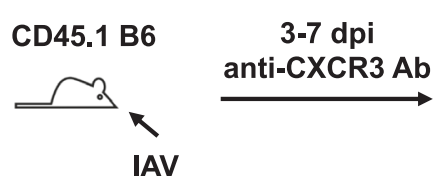

b

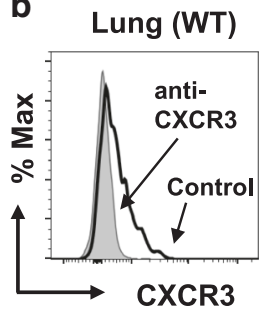

e

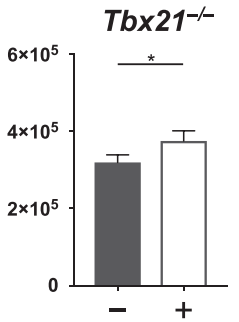

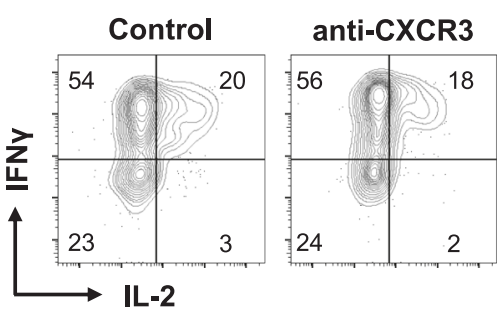

Fig. 5 CXCR3-mediated signals are required for maximal CD4 responses in the lung. a $1 \times 10^{6}$ of both naïve WT (Thy1.1/Thy1.2) and Tbx21 $1^{-1-}$ (Thy1.2) OT-II cells were transferred to WT CD45.1 $1^{+}$hosts, challenged with sublethal PR8-OAV ${ }_{11}$, and either treated with CXCR3 blocking antibody or PBS alone from 3-6 dpi. On 7 dpi, CXCR3 blockade was confirmed by FACS analysis assessing CXCR3 on WT OT-II cells using the same antibody clone, with (b) representative staining (c) and summary MFI analysis from WT donor cells 3 mice/group. $\mathbf{d}$ the absolute number of WT (left) and $\mathrm{Tb}_{2} \mathrm{1}^{-1-}$ (right) donor cells responding in the lung is shown. e Representative cytokine production from WT donor cells in the lung from mice either treated (right panel) or not (left panel) with CXCR3-blocking antibody. Results from one of three similar experiments

Improved IAV-primed memory generation from T-bet-deficient CD4 T cells

The ability of primed CD4 cells to contribute to immunity depends entirely on their ability to form memory. We thus tested how T-bet impacts memory generation following IAV priming. We first assessed expression of markers associated with differential survival potential of effector cells at the peak of the OT-II (7 dpi) and endogenous (8 dpi) CD4 T cell response. Tbx $21^{-1-}$ cells expressed reduced Ly6C (Fig. 7a), a marker of terminal differentiation in some models, ${ }^{24,25}$ and increased IL-7 receptor alpha chain (CD127) (Fig. 7b), increased levels of which correlate with improved fitness of effector CD4 T cells to form memory in this model. ${ }^{26} \mathrm{~Tb}^{2} \mathrm{1}^{-1-}$ effector cells also expressed more CD25 (Fig. 7c), consistent with their enhanced IL-2 production, and fitting with the need for IL-2 signaling to effector cells at this timepoint to induce sustained CD127. ${ }^{27}$

The phenotypic analysis above indicates that $T b \times 21^{-1-}$ cells may outcompete WT cells for memory niches. To test this in the most direct way, we transferred equal numbers of naïve WT (Thy1.1/Thy1.2) and Tbx21-/- (Thy1.2) OT-Il cells to CD45.1 ${ }^{+}$B6 hosts and primed with PR8-OVA $\|$ as in Fig. 5. We assessed the 

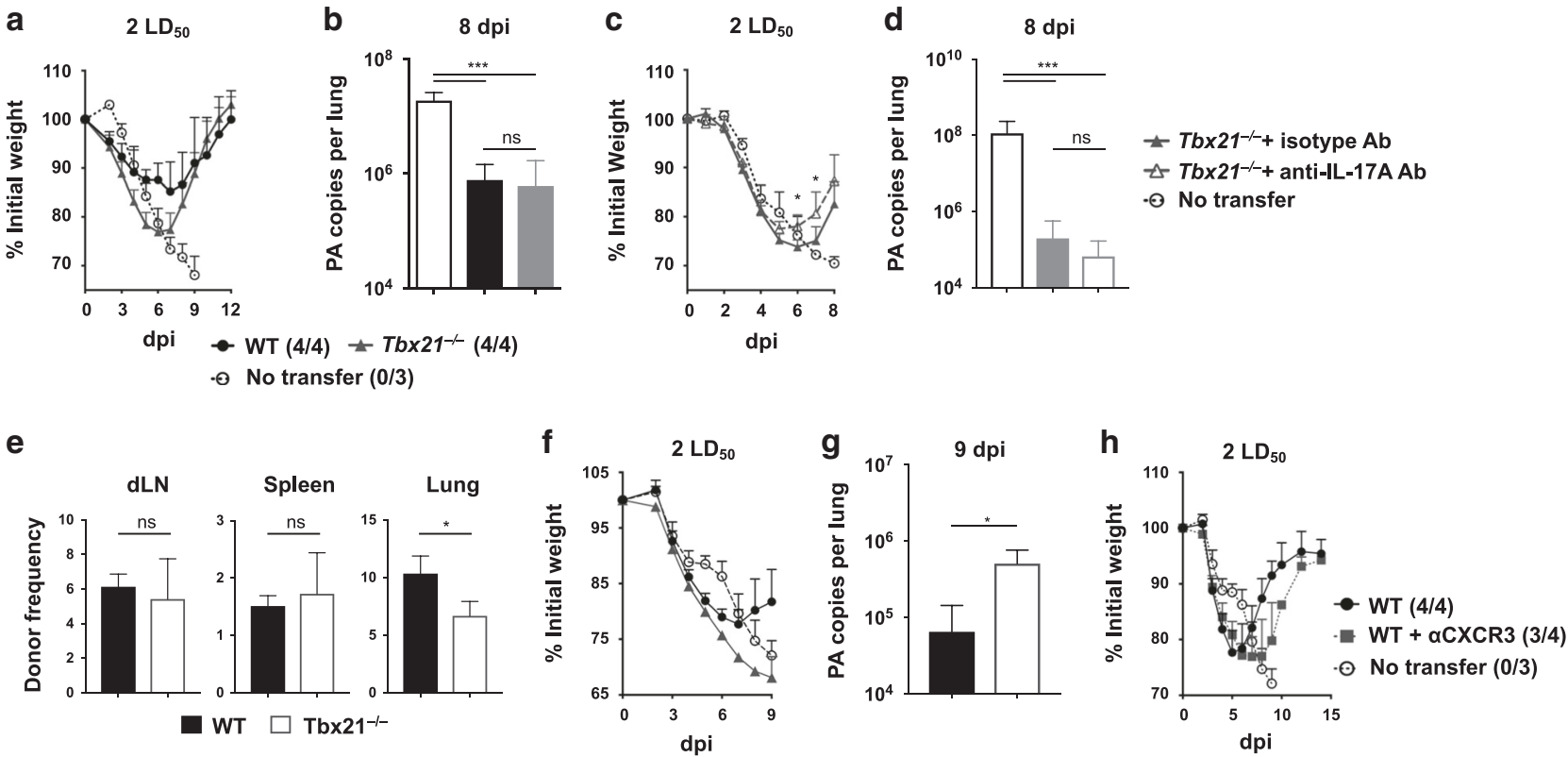

Fig. $6 \mathrm{~Tb} \times 21^{-/-}$effector cells mediate strong anti-viral responses against IAV. WT or Tbx21-/- OT-II cells were plated in Th1 conditions for 4 days to generate effectors. $3 \times 10^{6}$ effectors were transferred to naïve WT CD45.1 ${ }^{+}$hosts that were challenged with a lethal $2 L_{50}$ dose of PR8-OVA ${ }_{\| l}$. a Weight loss and recovery curves are shown from mice receiving effectors and control mice that did not receive cells $(n=4$ four for mice receiving effectors and $n=3$ for controls), with (b) viral titer analysis from groups of 3 mice at 7 dpi from a different experiment. Recipients of Tbx $21^{-1-}$ effector cells were challenged with 2 LD50 PR8-OVA neutralizing antibody or with an isotype control antibody. c Weight loss and recovery, and (d) viral titers at 7 dpi are shown. $\mathbf{e}$ The frequency of donor WT and Tbx $21^{-1-}$ effectors in stated organs is shown at 5 dpi. Adoptive hosts were given $1 \times 10^{6}$ effector cells and shown is the (f) weight loss and (g) pulmonary viral titer analysis from 4 mice/group at 9 dpi. Recipients of $3 \times 10^{6}$ WT effectors were treated with CXCR3blocking antibody from 0-7 dpi or with PBS alone. $\mathbf{h}$ Weight loss and recovery is shown from four mice/group. Results representative of at least two independent experiments

frequency of WT vs $T b \times 21^{-/-}$memory cells within the donor gate at $28 \mathrm{dpi}$, a well-accepted memory timepoint and an approach we have used previously to directly compare the fitness of CD4 cells with different genotypes to populate memory niches. ${ }^{26,28}$ Only about $20 \%$ of donor cells recovered at 28 dpi were WT, indicating a decisive advantage for Tbx $21^{-1-}$ cells to form memory and to survive long-term (Fig. $7 \mathrm{~d}$ ). Indeed, $\mathrm{Tb}_{2} 1^{-1-}$ cells continued to express higher CD127 than WT cells at 28 dpi (Fig. 7e), which we find is the most robust marker of CD4 T cell memory fitness in this model. ${ }^{26}$

We next re-stimulated the memory cells and assayed for IFN $\gamma$ and IL-17 and IL-2 production at $28 \mathrm{dpi}$ to determine the functional relationship of the $T b \times 21^{-1-}$ memory cells to the effector cells analyzed at $7 \mathrm{dpi}$. $T b \times 21^{-1-}$ cells produced reduced but still substantial IFN $\gamma$ vs. WT memory cells, retained a strong IL17 response component while virtually no IL-17 was detected from WT cells, and more $T b \times 21^{-1-}$ memory cells were $\mathrm{IL}-2^{+}$ (Fig. 7f). This analysis indicates that cytokine production potential of $\mathrm{Tb} \times 21^{-1-}$ cells as they transition from the effector to the memory state does not significantly shift in terms of prototypical Th1 vs Th17 functions.

Recent studies indicate that CD4 cells with reduced T-bet expression establish lung-resident memory after IAV priming with greater efficiency. ${ }^{29}$ In agreement with these findings, the majority of $T b \times 21^{-/-}$memory cells in the lung fit criteria of lung tissueresident memory by expression of a $\mathrm{CD} 69^{\text {high }} \mathrm{CD} 127^{\text {low }}$ phenotype, combined with shielding from labeling by fluorescent anti-CD4 antibody given to the mice just prior to organ harvest (Fig. 7g). In contrast, $T b \times 21^{-1-}$ memory cells in the spleen displayed a CD69 low $C D 127^{\text {high }}$ phenotype, consistent with our previous studies ${ }^{28}$ (Fig. $7 \mathrm{~g}$ ). Indeed, we observed similar high frequencies of CD69 high WT and Tbx $21^{-1-}$ OT-II memory cells in the lung at $28 \mathrm{dpi}$, and low frequencies of CD69 ${ }^{\text {high }}$ cells in the spleen (Fig. 7h). Virtually no FoxP ${ }^{+} \mathrm{CD} 25^{+}$memory cells were seen within either donor population (not shown), supporting results from Fig. 3 indicating that loss of T-bet does not promote Treg development. These results indicate that $T b \times 21^{-1-}$ cells outcompete WT CD4 T cells to populate both tissue-resident and conventional memory niches.

T-bet is not required for CD4 T cell-dependent heterosubtypic immunity against IAV

We next determined if T-bet expression is needed for IAV-primed mice to mount robust heterosubtypic immunity, which relies on IAV-specific memory T cells. We thus primed WT and Tbx $21^{-1-}$ mice with sublethal PR8 (H1N1) and challenged at 28 dpi with A/ Philippines (H3N2) to test if T-bet expression, even by non-T cells, contributes to recovery against primary or secondary infection. When primed with $0.5 \mathrm{LD}_{50} \mathrm{PR} 8, \mathrm{WT}$ and $\mathrm{Tb} \times 21^{-/-}$mice lost equivalent weight and began to recover on the same dpi (Fig. 8a). Histological analysis revealed no clear distinctions in lung pathology at 7 dpi (Fig. 8b), and viral control was equivalent (Fig. 8c). Total PR8-specific IgG levels were similar in WT and $\mathrm{Tb} \times 21^{-1-}$ mice. Given that high titers of long-lived IgG in IAVprimed mice require $T_{F H}$ help, ${ }^{30}$ this indicates similar $T_{F H}$ activity in $\mathrm{WT}$ and $\mathrm{Tb} \times 21^{-1-}$ mice, in agreement with analysis in Fig. 3. IgG2a production was impaired in $T b \times 21^{-1-}$ mice while $\operatorname{lgG} 1$ was enhanced (Fig. $8 \mathrm{~d}$ ). To test the protective capacity of the antiPR8 serum generated in WT or Tbx $21^{-1-}$ mice, we passively transferred $50 \mu \mathrm{L}$ of immune serum to naive WT mice that were then challenged with $10 \mathrm{LD}_{50}$ PR8 as in a previous study. ${ }^{30}$ Recipients of WT or Tbx $21^{-1-}$ serum were protected from lethal challenge while controls succumbed by $10 \mathrm{dpi}$ (Fig. 8e). As protection in this model requires $\mathrm{T}_{\mathrm{FH}}$-dependent titers of PR8specific lgG, ${ }^{30}$ this indicates similar protective quality of CD4 T celldependent humoral immunity in the WT and $T b \times 21^{-1-}$ mice.

We next re-challenged the PR8-primed mice at $28 \mathrm{dpi}$ with a

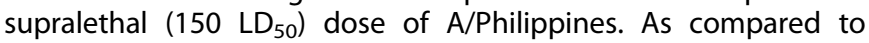


a

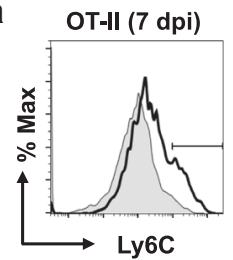

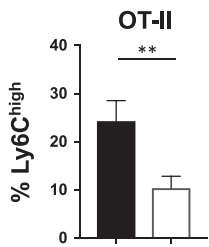

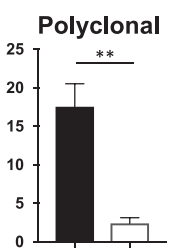

b
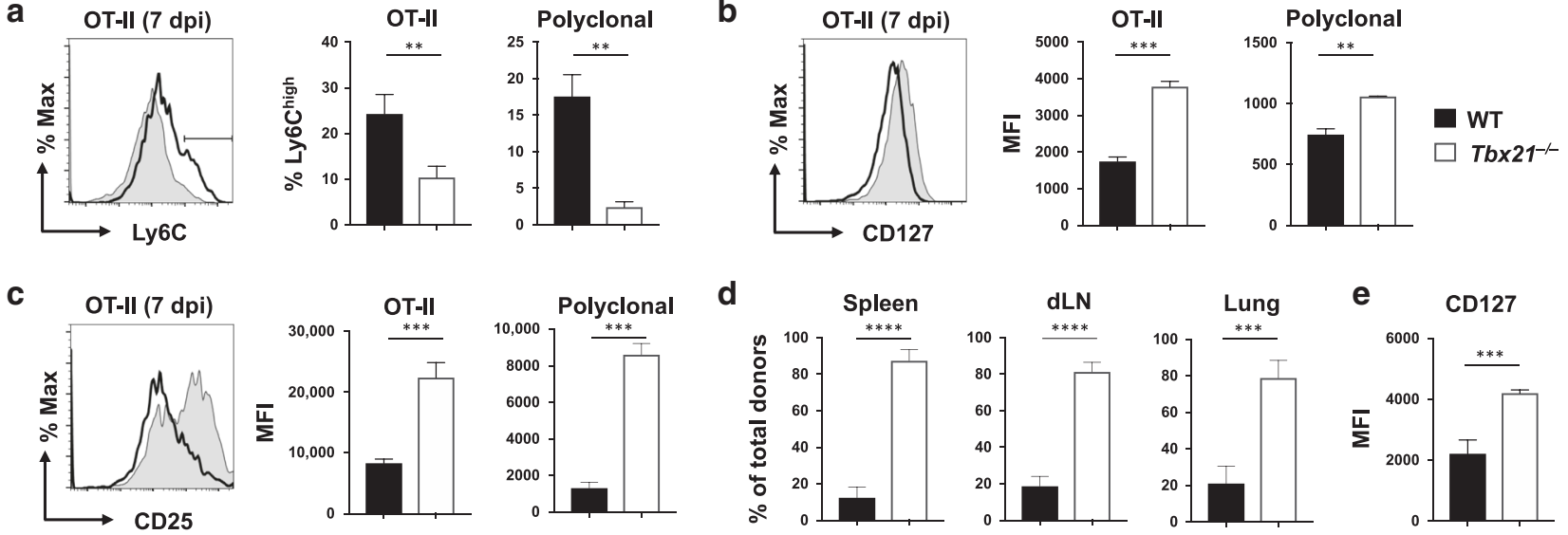

d
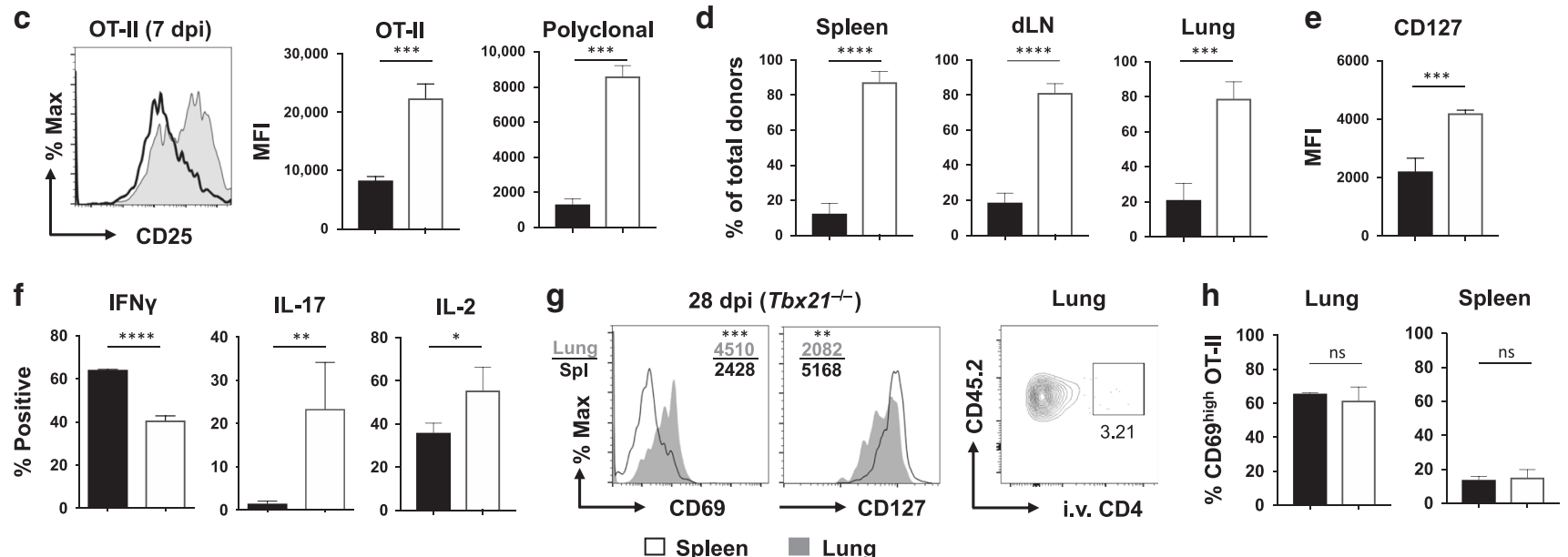

Fig. 7 Tbx21 $1^{-1-}$ CD4 cells outcompete WT cells for memory niches following IAV priming. Representative staining (left, histogram) and summary analysis of lung effectors from groups of 4 mice receiving OT-II donor cells at $7 \mathrm{dpi}$ (left bar graph) or from analysis of NP ${ }_{311}^{+}$polyclonal cells responding in bone marrow chimeras at 8 dpi from 3 mice per group (right bar graph) for (a) Ly6C, (b) CD127, and (c) CD25. $1 \times 10^{6}$ naïve WT

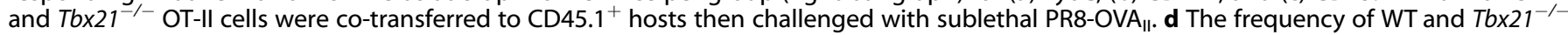
cells within the donor gate at $28 \mathrm{dpi}$ (4 mice/group). e The MFI of CD127 expression by lung donor cells is shown at $28 \mathrm{dpi}$ from 4 mice/group. f Summary analysis of IFN $\gamma, \mathrm{IL}-17$, and IL-2 production from co-transferred WT and Tbx $21^{-1-}$ OT-II cells in the lungs at 28 dpi from 4 mice. At 28 dpi, adoptive hosts were given fluorescent anti-CD4 antibody i.v. and organs harvested 5 min later. g Shown is representative staining of Tbx $21^{-1-}$ donor cells in the spleen (open) and lung (shaded) for CD69 (left histogram) and CD127 (right histogram), as well as lung for donor cell protection from the i.v. administered anti-CD4 antibody. $\mathbf{h}$ The frequency of CD69 high WT (black) and Tbx21 ${ }^{-1-}$ (open) OT-Il cells in the lung and spleen is shown at $28 \mathrm{dpi}$ from 3 mice/group. Results representative of two independent experiments

unprimed WT controls that lost $\sim 30 \%$ of their weight, primed WT and $T b \times 21^{-1-}$ mice lost minimal weight and recovered by 7 dpi (Fig. 8f). Analysis of viral titer revealed equivalent control in both strains of PR8-primed mice (Fig. 8g). Because memory CD8 cells might play a more dominant role in the $\mathrm{Tb} \times 21^{-/-}$vs. WT mice, potentially masking deficient CD4 cell-dependent immunity, we depleted CD8 T cells in PR8-primed mice prior to A/Philippines infection. All primed mice were protected while controls rapidly succumbed (Fig. 8h), indicating that T-betdeficient memory CD4 cell-mediated protection, even in the absence of CD8 memory, is sufficient to clear even supralethal heterosubtypic IAV challenges.

Analysis of CD4 $\mathrm{T}$ cells responding in the bronchoalveolar lavage (BAL) of PR8-primed mice challenged with A/Philippines revealed similar patterns of decreased CXCR3 and Ly6C, but increased CD25 expressed by $T b \times 21^{-1-}$ cells (Fig. 8i). The BALresident $T b \times 21^{-1-}$ CD4 T cells produced much reduced levels of IFN $\gamma$ but increased IL-17 vs. WT cells (Fig. 8j). These results demonstrate conserved hallmarks of protective Tbx21-/- CD4 T cell responses against IAV during primary and recall responses against IAV.

Finally, to test if T-bet differentially impacts key protective phases of the recall response, we transferred WT or Tbx $21^{-1-}$ OT-II memory CD4 T cells generated in Th1-polarizing conditions to naive WT hosts and challenged them with lethal PR8-OVA $A_{\text {II }}$. These populations mirror the responses of polyclonal memory CD4 T cells generated by IAV-priming, and we have used this model to identify discrete phases of memory CD4 T cell protection. 3,16,26,31 WT or $\mathrm{Tb} \times 21^{-/-}$memory cells protected otherwise naive hosts (Fig. 8k), in agreement with the results in Fig. 8h of intact CD4 T cell-dependent immunity in IAV-primed mice. Protection in this model involves an early phase, in which the memory cells induce an accelerated innate immune response to control viral titers within $4 \mathrm{dpi}^{32}$ and a late phase that requires the development of large numbers of $2^{\circ}$ effectors from the memory cells to control titers at $7 \mathrm{dpi}^{16}$ At both 4 and $7 \mathrm{dpi}$, viral titers were similarly controlled in recipients of WT and Tbx $21^{-1-}$ memory cells vs. titers in control mice not receiving donor cells (Fig. 8l). These results indicate that neither the 'jump-starting' of protective innate immunity in the lung by memory cells, nor the generation of antiviral secondary effectors requires CD4 cell-intrinsic T-bet, and provide further evidence that highly protective primary and secondary CD4 T cell responses against IAV do not require T-betdependent programming.

\section{DISCUSSION}

Defining the attributes of CD4 T cells needed to combat specific pathogens is critical to improve the ability of vaccines to induce protective cellular immunity. The clearance of intracellular pathogens is strongly linked with responses orchestrated by Th1 cells that require T-bet for their programming. Indeed, outcomes of Leishmania donovani, ${ }^{33}$ Toxoplasma gondii, ${ }^{34}$ Mycobacterium tuberculosis, ${ }^{35}$ Mycobacterium avium, ${ }^{36}$ Staphylococcus aureus, ${ }^{37}$ Salmonella typhimurium, ${ }^{38}$ Francisiella tularensis, ${ }^{39}$ and Trypanosoma $\mathrm{Cruzi}^{40}$ infection are all more severe in $\mathrm{Tb} \times 21^{-1-}$ vs. WT mice. Similarly, T-bet is required for optimal protection against Herpes Simplex virus $2^{41}$ and Vaccinia, ${ }^{42}$ Rabies, $^{43}$ and while not required to clear Rhinovirus, infected $T b \times 21^{-/-}$mice develop severe asthma-like inflammation. ${ }^{44}$ It is thus surprising that even 


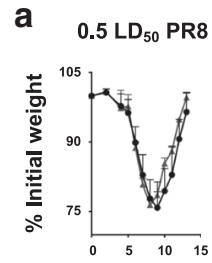

dpi

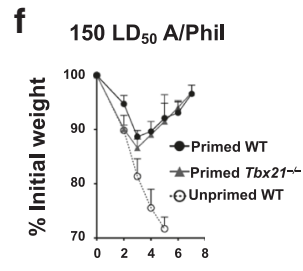

dpi

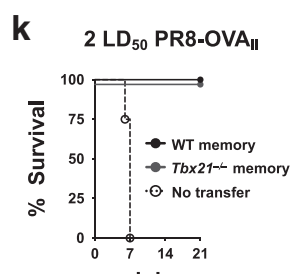

dpi
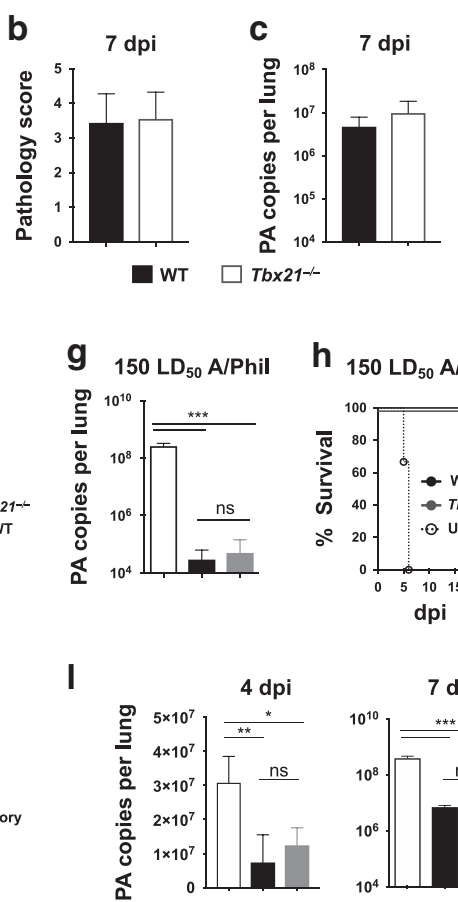

$h_{150 L D_{50}}$ A/Phil

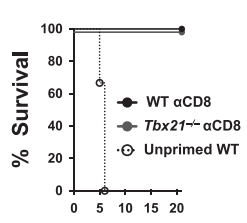

dpi

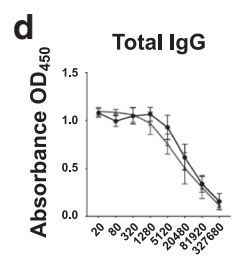

Serum dilution

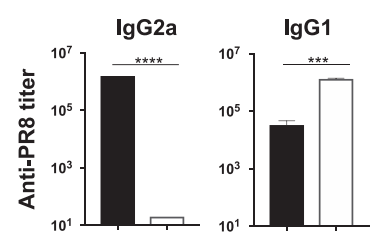

I 4 dpi BAL $\left(\mathrm{CD} 90^{+} \mathrm{CD}^{+}\right.$gate $)$

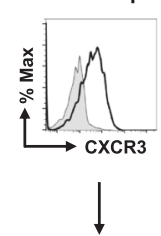

CXCR3

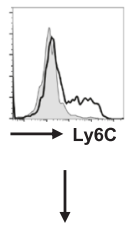

Ly6C

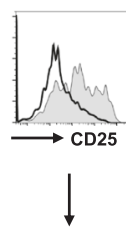

CD25
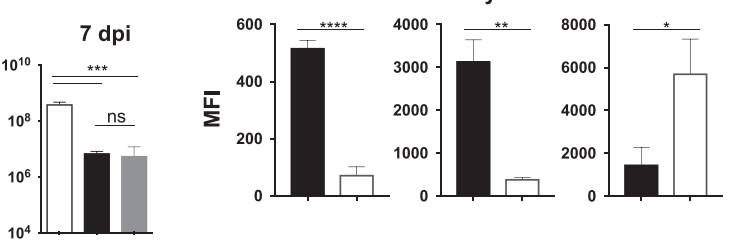

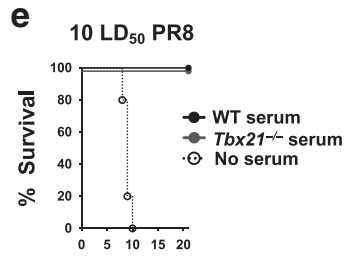

dpi
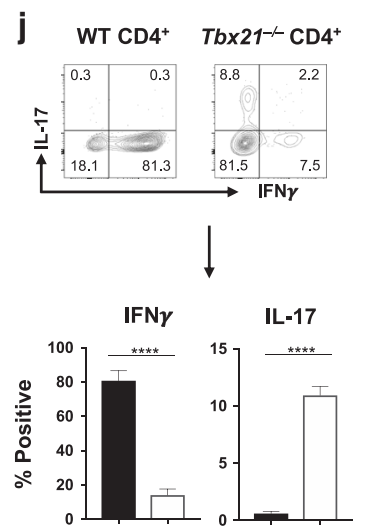

Fig. $8 \mathrm{~Tb} \times 21^{-1-}$ mice are protected against primary and secondary IAV challenge. WT and Tbx21 $1^{-/-}$B6 mice were challenged with 0.5 LD ${ }_{50}$ PR8. Shown is (a) weight loss and recovery, (b) average pathology scores from 7 dpi, and (c) viral titers at 7 dpi from groups of $3-4$ mice. At 21 dpi, serum was harvested from mice infected with 0.5 LD $_{50}$ PR8 and analyzed for total PR8-specific IgG. Shown is the (d) average OD reading from

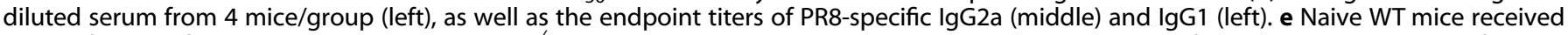
$50 \mu \mathrm{L}$ of serum from PR8-primed WT or Tbx21-1- mice and were challenged with 10 LD $_{50}$ PR8. Survival from 3 mice per group of serum recipients is shown vs. 5 control mice not receiving serum. Mice primed as in (a) were challenged with a A/Phil 28 days later. Shown is (f) weight loss and recovery for groups of 4 PR8-primed WT and Tbx21 $1^{-1}$ mice vs. 3 unprimed WT mice and (g) viral titers from 8 dpi. PR8-primed mice were treated with CD8-depleting antibody prior to heterosubtypic challenge. (h) Survival is shown for groups of 4 primed mice and 3 unprimed controls. Representative of three independent experiments. BAL cells were harvested from A/Phil-challenged PR8-primed mice and $\mathrm{CD}{ }^{+} \mathrm{CD}^{+}$cells analyzed for surface markers (i) and cytokine production (j) (3 mice per group). In separate experiments, unprimed WT mice received $5 \times 10^{6}$ WT or Tbx $21^{-1-}$ memory OT-II cells and were challenged with PR8-OVA II. $^{-k}$ Survival from four mice per condition is shown along with (I) analysis in separate experiments of viral titers on $4 \mathrm{dpi}$ (left) and 7 dpi (right) (4 mice per group with cell transfer and 3 mice per group without cell transfer). Results representative of two similar experiments

though CD4 T cells primed by IAV express high levels of T-bet, Tbet is not required for efficient clearance of primary or heterosubtypic IAV infection.

Although many changes in cytokine production distinguish WT and $T b \times 21^{-1-}$ CD4 cells responding to IAV, IFNY is still the predominant cytokine made by $T b \times 21^{-1-}$ cells, albeit at reduced levels vs. WT effectors. STAT4-dependent signals may be sufficient to direct this residual IFN $\gamma$ production, a hypothesis supported by some IL-12-dependent IFNy seen from Tbx21 $1^{-/-}$effectors in "Th1" conditions in vitro. A non-mutually exclusive possibility is that Eomesodermin (Eomes) can substitute for T-bet. Eomes has been shown to direct robust IFNy production in CD4 cells, but in the absence of T-bet, the cells can gain Th17 function. ${ }^{45}$ Indeed, we observed a sizable IL- $17^{+}$and Roryt ${ }^{+}$subset within $T b \times 21^{-1-}$ but not WT cells primed by IAV, consistent with a potential role for Eomes, and consistent with T-bet's role in suppressing Th17 development. ${ }^{46}$ Further studies are required to determine the importance of STAT4 and Eomes in directing the development of effective CD4 T cell responses. It will also be important to elucidate the full extent of changes in gene expression patterns between WT and $T b \times 21^{-1-}$ effectors responding to IAV in order to reveal phenotypic and functional distinctions beyond those highlighted in this study, and to test the roles of any such genes in contributing to the protective CD4 T cell response against IAV. Such analysis is crucial in order to identify novel correlates of protective CD4 $\mathrm{T}$ cell responses beyond traditional measures of strong Th1 polarization.
Increased IL-17 production was the most remarkable gain of function in the $T b \times 21^{-/-}$vs. WT CD4 T cells responding to IAV in terms of cytokine production. However, we found IL-17 neutralization not to markedly impact protection mediated by $T b \times 21^{-1-}$ effectors. Whether other aspects of Th17 programming underlie the ability of $T b \times 21^{-/-}$CD4 T cells to combat IAV remains to be determined, but this possibility is suggested by a significant population of Roryt ${ }^{+} \mathrm{Tb} \times 21^{-/-}$effector cells detected in the lungs of IAV-infected mice.

Decreased levels of the chemokine receptor CXCR3 characterized the most striking phenotypic distinction between $T b \times 21^{-1-}$ and WT CD4 T cells responding to IAV. Furthermore, high CXCR3 expression is the most relevant T-bet-dependent correlate of protective CD4 cells identified herein. This conclusion is similar to recent findings of impaired Toxoplasma gondii control in peripheral tissues of $T b \times 21^{-1-}$ vs. WT mice that was associated with reduced $T$ cell accumulation with less CXCR3. ${ }^{34}$ We note, however, that CXCR3 blockade only decreased WT effectors in the lungs by about one half vs. the 5-10-fold decrease seen in CXCR3deficient CD4 cell lung trafficking during Sendia virus infection. ${ }^{18}$ This reflects strong CXCR3-independent recruitment operating during IAV infection. IAV drives not only CXCL9 and CXCL10 (ligands for CXCR3) but also CCL2, ${ }^{32}$ a ligand for CCR4, which was increased on $\mathrm{Tb} \times 21^{-1-}$ cells, as well as several other major chemokines. Multiple layers of redundancy may thus provide for CXCR3-independent CD4 trafficking sufficient to control even higher doses of IAV. A similar explanation may at least in part 
underlie intact immunity against LCMV and Listeria monocytogenes reported in $T b \times 21^{-1-}$ mice. ${ }^{47,48}$

T-bet-deficient CD4 effector cells outcompete WT cells for memory after IAV priming. Our results are consistent with studies linking higher T-bet and Ly6C expression by CD4 effector cells responding to $\mathrm{LCMV}^{24}$ and to murine $\gamma$-herpesvirus $68^{25}$ with a terminal fate. The $T b \times 21^{-/-}$effectors primed by IAV also express higher CD127. CD127 is positively regulated by IL-2 during IAV responses, ${ }^{26}$ suggesting that enhanced IL-7 receptor expression by $T b \times 21^{-1-}$ cells may be linked to their increased production of IL-2 vs. WT cells. While our findings support work indicating that Tbet restricts lung-resident CD4 memory potential, ${ }^{29}$ we also saw improved $T b \times 21^{-1-}$ memory in the spleen and dLN. In contrast to the IL-7-dependence of memory homeostasis in secondary lymphoid organs, survival of at least some lung-resident CD4 memory is IL-7-independent. ${ }^{28}$ T-bet expression may thus restrict both canonical and non-canonical pathways impacting diverse memory subsets primed by IAV. It is interesting to speculate that T-bet-dependent control of the glycolysis pathway in CD4 T cells ${ }^{49}$ may contribute differential memory fitness of WT and Tbx $21^{-1-}$ effector cells, given the prime role for cellular metabolism that is emerging in the governance of $\mathrm{T}$ cell memory fates. ${ }^{50} \mathrm{~A}$ full investigation of how T-bet impacts metabolic programming of IAV-primed effector and memory CD4 T cell subsets may provide novel targets for vaccines to improve the establishment and durability of cellular immunity.

T-bet-deficient mice retain the ability to clear even high doses of primary IAV infection as well as WT mice. These results are in agreement with a recent study ${ }^{51}$ finding low-dose IAV-primed $T b \times 21^{-1-}$ mice are better protected against secondary bacterial infection than are WT animals. We extend these observations to show that IAV-primed $T b \times 21^{-1-}$ mice develop robust heterosubtypic immunity with no discernable defects compared to WT mice following supralethal IAV challenges. While the focus of this study is on how CD4 T cell-intrinsic T-bet expression impacts this subset's response potential, our limited experiments analyzing primary and heterosubtypic IAV challenge in WT and Tbx $21^{-1-}$ provide important insights into T-bet's roles in regulating other aspects of the IAV-immune state. First, as the experiments assessing heterosubtypic immunity were done in full T-betdeficient animals, our findings indicate neither adaptive nor innate immune cells require T-bet expression to clear primary or secondary IAV infection. Our results cannot determine if the role of individual innate immune subsets gain or lose importance in contributing to viral control in the absence of T-bet. However, they do indicate that T-bet induction is not prerequisite to program protective "trained innate immunity" that can synergize with memory $T$ cell responses to clear even supralethal heterosubtypic IAV challenge in primed mice. Second, our data indicates that virus-specific lgG2a is not a required element of heterosubtypic immunity, which is of interest in gaining further understanding of the roles of non-neutralizing antibodies in contributing to vaccineinduced protection against IAV. That similar titers of PR8-specific lgG were detected in WT and Tbx $21^{-1-}$ mice is indicative of intact $\mathrm{T}_{\mathrm{FH}}$ activity, as virus-specific IgG in IAV-primed C57BL/6 mice lacking $T_{F H}$ is reduced by 1 to 2 logs vs. WT mice. ${ }^{30}$ Indeed, $T_{F H}$ were detected at equal frequencies within WT and $T b \times 21^{-1}$ cells responding to IAV in WT hosts. Recent observations indicate that $\mathrm{T}_{\mathrm{FH}}$ may contain subsets that mirror conventional effector states (i.e., Th1, Th2, Th17). ${ }^{52}$ Given this framework, reduced lgG2a but increased $\lg \mathrm{G} 1$ in $\mathrm{Tb} \times 21^{-1-}$ mice may reflect decreased $\mathrm{IFNY}^{+}$ "Th1" $\mathrm{T}_{\mathrm{FH}}$ and increased IL-4 "Th2" $\mathrm{T}_{\mathrm{FH}}$. However, B cells can also express T-bet with important functional consequences. ${ }^{53}$ Further studies are thus required for a full understanding of how T-bet regulates humoral immunity against IAV.

In summary, our studies indicate that T-bet-dependent programming is not required to generate effector or memory CD4 cells with strong anti-viral functions. However, upregulation of CXCR3, which is T-bet-dependent, is needed to maximize the efficacy of CD4 responses against IAV by promoting accumulation of effector cells in the infected lung. Finally, our results indicate that the goal of promoting strong Th1 responses against IAV may limit the ability of such effector cells to form protective memory. Our results indicate the need to better characterize and differentiate CD4 cells that are defined as "Th1" based on IFNy production alone. Such efforts will help to uncover more decisive correlates of protective cells in specific disease settings and also allow for the development of novel vaccine strategies that induce specific attributes in effector cells best suited for responses against specific pathogens à la carte rather than through sweeping polarization programs.

\section{METHODS}

Mice

C57BL6 (B6) mice knocked out for T-bet $\left(\mathrm{Tb} \times 21^{-/-}\right)$or wild-type B6.CD45.1 mice were used at least 8 weeks old for IAV infection. Donor CD4 cells for adoptive transfer experiments were obtained from 4-8 week old OT-II TCR transgenic mice on a WT (Thy1.1/ Thy1.2) or $T b \times 21^{-/-}$(Thy1.2) background. The OT-II TcR recognizes aa 323-339 of chicken ovalbumin (OVA). For bone marrow chimeras, B6.Thy1.1 mice were lethally irradiated and reconstituted intravenously with $2 \times 10^{6} \mathrm{~T}$ and $\mathrm{B}$ cell-depleted bone marrow cells from B6.CD45.1 or T-bet ${ }^{-1-}$ mice. Reconstitution was verified by FACS analysis of peripheral blood prior to use in experiments. All mice were originally obtained from Jackson Laboratories (Bar Harbor, ME) and bred at the University of Central Florida. All experimental animal procedures were approved by and conducted in accordance with the University of Central Florida's Animal Care and Use Committee's guidelines.

CD4 T cell isolation, effector and memory generation, and cell transfer

Naïve $\mathrm{CD}^{+}$cells from OT-II donor mice were obtained from pooled spleen and lymph nodes of unmanipulated mice. Single cell suspensions were incubated on nylon wool for one hour followed by Percoll gradient separation and then positive MACS selection using CD4 microbeads (Miltenyi Biotec, Auburn, CA). The resulting cells were routinely $>97 \% \mathrm{TCR}^{+}$and expressed a naïve phenotype (CD62L ${ }^{\text {high }}, C$ 44 $\left.{ }^{\text {low }}\right)$. Naïve CD4 cells were used to generate effector cells in vitro, or used for adoptive transfer experiments.

Effector cells were generated as previously described ${ }^{13,54}$ using irradiated T-depleted spleen cells as APC and OVA Briefly, all culture conditions were supplemented with IL-2 at $11 \mathrm{ng} / \mathrm{mL}$; Th1 cultures were further supplemented with anti-IL-4 antibody (clone 11B11) at $15 \mathrm{ug} / \mathrm{mL}$ and $\mathrm{IL}-12$ at $2 \mathrm{ng} / \mathrm{mL}$; Th2 cultures were further supplemented with anti-IFNy antibody (clone XMG1.2) at $15 \mathrm{ug} / \mathrm{mL}$, and IL-4 at $15 \mathrm{ng} / \mathrm{mL}$; Th17 cultures were further supplemented with anti-IL-4 and anti-IFNY both at $15 \mathrm{ug} / \mathrm{mL}, \mathrm{IL}-6$ at $20 \mathrm{ng} / \mathrm{mL}, \mathrm{IL}-23$ at $25 \mathrm{ng} / \mathrm{mL}, \mathrm{IL}-21$ at $50 \mathrm{ng} / \mathrm{mL}$, TGF $\beta$ at $0.5 \mathrm{ng} / \mathrm{mL}, \mathrm{IL}-1$ at $10 \mathrm{ng} / \mathrm{mL}$, TNF at $10 \mathrm{ng} / \mathrm{mL}$. All blocking antibodies were purchased from BioXcell (West Lebanon, NH). All other reagents were purchased from Peprotech (Rocky Hill, NJ). Effector cultures were fed with fresh media and IL-2 at 2 days, and the resulting effector cells were analyzed at 4 days. Effectors were thoroughly washed prior to adoptive transfer experiments. Memory populations were generated and assessed as previously described ${ }^{54}$ by thoroughly washing effector cells, resting the cells for at least 3 days in fresh media without added cytokines or peptide, followed by isolation of live cells using lympholyte $M$ (Cedarlane, Burlington, NC).

Naïve, effector, and memory CD4 cells were adoptively transferred to host mice under light anesthesia in $200 \mu \mathrm{L}$ of RPMI media alone via retro-orbital injection. 
Viral stocks and infections and in vivo antibody treatments A/PR8 and A/PR8-OVA $A_{\|}(\mathrm{H} 1 \mathrm{~N} 1)$ were grown in the allantoic cavity of embryonated hen eggs from stocks originally provided by $P$. Doherty. A/Philippines/2/82/x-79 (H3N2) was similarly prepared from stocks originally provided by S. Epstein. All viral stocks were characterized at the Trudeau Institute (Saranac Lake, NY). Virus was administered to mice under light isoflurane anesthesia intranasally in $50 \mu \mathrm{L}$ of PBS. All infected mice were monitored daily for weight loss, hunched posture, ruffled fur, and lack of movement and euthanized when humane endpoints were reached.

In some experiments, mice were treated with $250 \mu \mathrm{g}$ of neutralizing antibody against IL-17 (clone 17F3) administered by intraperitoneal injection on days $-1,1,3,5$, and 7 post IAV infection. In other experiments, mice were treated with $250 \mu \mathrm{g}$ blocking antibody against CXCR3 (clone BE0249) by intraperitoneal injection daily throughout the first week of IAV infection. All antibodies used to treat mice were purchased from BioXcell (West Lebanon, $\mathrm{NH}$ ).

In some experiments, $50 \mu \mathrm{L}$ of serum obtained from PR8-primed mice at 28 dpi was transferred to naive WT mice by intraperitoneal injection. Two hours later, the mice were challenged with 10 LD50 PR8.

\section{Tissue preparation}

At different time points after virus infection, mice were euthanized by cervical dislocation followed by exsanguination by perforation of the abdominal aorta. Lungs, spleen, and draining mediastinal lymph node (dLN) were prepared into single cell suspensions by mechanical disruption of organs and passage through a nylon membrane.

For assessment of immunopathology following IAV infection, lung lobes were isolated and immediately fixed in 10\% neutral buffered formalin. Lung samples were subsequently processed, embedded in paraffin, sectioned, placed on L-lysine-coated slides, and stained with Hematoxylin and Eosin (H\&E) using standard histological techniques. The extent of mononuclear cell infiltration and tissue damage were graded blindly from 0 to 4 by a boardcertified pathologist (S. Sell) as described previously. ${ }^{55}$

In some experiments BAL was harvested by gently instilling PBS into the bronchioles followed by gentle retraction using a syringe and catheter. Two installations and retractions of $1 \mathrm{~mL}$ were performed to collect airway-resident cells.

The level of IAV-specific antibody (total lgG and lgG2a) was determined in convalescent sera obtained from A/PR8-primed mice at $21 \mathrm{dpi}$ by ELISA as previously described. ${ }^{30}$

\section{Flow cytometry}

Single-cell suspensions were washed, resuspended in FACS buffer (PBS plus $0.5 \%$ BSA and $0.02 \%$ sodium azide) and incubated on ice with $1 \mu \mathrm{g}$ of anti-FcR (2.4G2) and optimized concentrations of the following fluorochrome-labeled antibodies for surface staining: anti-Thy1.1 (OX-7), anti-Thy1.2 (53-2.1), anti-CD4 (RM4.5), antiCD69 (H12F3), anti-CD25 (PC61.5), anti-CD127 (A7R34), anti-CD44 (IM7), anti-CD45.2, anti-CXCR3 (CXCR3-173), anti-CCR4 (2G12), antiLy-6C (HK1.4), anti-CCR7 (4B12), anti-CD44 (IM7), anti-CCR6 (29-2L17), anti-CCR5 (HM-CCR5), anti-CXCR5 (SPRCL5), anti-CD11a (M17/4), and anti-PD1 (J43). To detect IAV-specific polyclonal CD4 cells, cell suspensions were stained for $1 \mathrm{~h}$ (hr.) at $37^{\circ} \mathrm{C}$ with $\mathrm{I}-\mathrm{A}^{\mathrm{b}} /$ $\mathrm{NP}_{311-325}$-fluorochrome-labelled tetramer obtained from the $\mathrm{NIH}$ tetramer facility (Atlanta, GA) prior to surface marker staining.

For intracellular cytokine staining cells were stimulated for $4 \mathrm{~h}$ with $10 \mathrm{ng} / \mathrm{ml}$ PMA and $50 \mathrm{ng} / \mathrm{ml}$ ionomycin and $10 \mu \mathrm{g} / \mathrm{ml}$ Brefeldin A added after $2 \mathrm{~h}$. Cells were then surface stained and fixed for $20 \mathrm{~min}$ in $4 \%$ paraformaldehyde followed by permeablization for 10 min by incubation in $0.1 \%$ saponin buffer (PBS plus $1 \%$ FBS, $0.1 \%$ NaN3 and $0.1 \%$ saponin) and then stained for cytokine by the addition of anti-IFN- $\gamma$ (XMG1.2), anti-IL-2 (JES65H4), anti-TNF (MP6-XT22), anti-IL-17 (TC11-18H10.1), anti-IL-10
(JES5-16E3), anti-GM-CSF (MP1-22E9), and anti-Granzyme B (NGZB) fluorescently labeled antibodies for $20 \mathrm{~min}$.

Detection of transcription factors and Ki67 by flow cytometry was conducted using intranuclear staining protocols staining as per manufactures' (ThermoFisher) instructions with fluorescently labeled antibodies against T-bet (Ebio4B10), Roryt (B2D), GATA-3 (TWAJ), Eomes (Dan11mag), and FoxP3 (3G3), and Bcl-6 (BCLDWN).

All FACS analysis was performed using a Canto (BD Biosciences) or Cytoflex (Beckman Coulter) flow cytometers and FlowJo (Tree Star) analysis software. All antibodies were purchased from BD Biosciences (San Jose, CA), Biolegend (San Diego, CA), or Thermo Fisher (Waltham, MA).

\section{Real time-PCR}

Viral titers were determined by quantitation of viral RNA prepared from whole lung homogenates using TRIzol (Sigma-Aldrich). $2.5 \mu \mathrm{g}$ of RNA was reverse transcribed into CDNA using random hexamer primers and Superscript II Reverse Transcriptase (Invitrogen). Quantitative PCR was performed to amplify the polymerase (PA) gene of PR8 and A/Phil using an ABI Prism 7700 Sequence Detector (Applied Biosystems) with $50 \mathrm{ng}$ of cDNA per reaction and the following primers and probe: forward primer, 5'CGGTCCAAATTCCTGCTGA-3'; reverse primer, 5'CATTGGGTTCCTT CCATCCA-3'; probe, 5'-6-FAM-CCAAGTCATGAAGGAGAGGGA ATACCGCT-3'. Data were analyzed with Sequence Detector v1.7a (Applied Biosystems). The copy number of the PA gene per $50 \mathrm{ng}$ of CDNA was calculated using a PA-containing plasmid of known concentration as a standard.

\section{Statistical analysis}

Unpaired, two-tailed, Students $t$-tests, $\propto=0.05$, were used to assess whether the means of two normally distributed groups differed significantly. The Welch-correction was applied when variances were found to differ. One-way ANOVA analysis with Bonferroni's multiple comparison post-test was employed to compare multiple means. Significance is indicated as ${ }^{*} P<0.05$; ${ }^{* *} P<0.005$; ${ }^{* *} P<$ 0.001 ; and ${ }^{* * * *} P<0.0001$. The Log Rank test was used to test for significant differences in Kaplan-Meier survival curves. All error bars represent standard deviation.

\section{ACKNOWLEDGEMENTS}

We thank the NIH Tetramer Core Facility for providing the $\mathrm{NP}_{311-235}$ tetramer and control reagents. We thank the University of Central Florida's Vivarium staff for providing excellent care for the animals in this study. We thank Dr. Priyadharshini Devarajan for helpful discussions. This work was supported by American Heart Association grant 14SDG18600020 (to K.K.M.), National Institutes of Health Grant Al117457 (to T.M.S.) and by funds provided by the University of Central Florida.

\section{AUTHOR CONTRIBUTIONS}

K.D. and C.F. performed all experiments. K.D. and K.K.M. analyzed data and wrote the manuscript. S.S. performed blinded analysis of histopathology. T.M.S. provided key reagents, reviewed and critiqued the manuscript and figures, and contributed to interpretation of the findings.

\section{ADDITIONAL INFORMATION}

The online version of this article (https://doi.org/10.1038/s41385-019-0183-z) contains supplementary material, which is available to authorized users.

Competing interests: The authors declare no competing interests.

Publisher's note: Springer Nature remains neutral with regard to jurisdictional claims in published maps and institutional affiliations. 


\section{REFERENCES}

1. Swain, S. L., McKinstry, K. K. \& Strutt, T. M. Expanding roles for CD4( + ) T cells in immunity to viruses. Nat. Rev. Immunol. 12, 136-148 (2012).

2. Szabo, S. J. et al. A novel transcription factor, T-bet, directs Th1 lineage commitment. Cell 100, 655-669 (2000).

3. McKinstry, K. K. et al. Memory CD4 + T cells protect against influenza through multiple synergizing mechanisms. J. Clin. Invest 122, 2847-2856 (2012).

4. Strutt, T. M. et al. Multipronged CD4( +) T-cell effector and memory responses cooperate to provide potent immunity against respiratory virus. Immunol. Rev. 255, 149-164 (2013).

5. Graham, M. B., Braciale, V. L. \& Braciale, T. J. Influenza virus-specific CD4 +T helper type $2 \mathrm{~T}$ lymphocytes do not promote recovery from experimental virus infection. J. Exp. Med. 180, 1273-1282 (1994).

6. Brown, D. M., Dilzer, A. M., Meents, D. L. \& Swain, S. L. CD4 T cell-mediated protection from lethal influenza: perforin and antibody-mediated mechanisms give a one-two punch. J. Immunol. 177, 2888-2898 (2006).

7. Teijaro, J. R., Verhoeven, D., Page, C. A., Turner, D. \& Farber, D. L. Memory CD4 $T$ cells direct protective responses to influenza virus in the lungs through helperindependent mechanisms. J. Virol. 84, 9217-9226 (2010).

8. Brown, D. M., Lee, S., Garcia-Hernandez Mde, L. \& Swain, S. L. Multifunctional CD4 cells expressing gamma interferon and perforin mediate protection against lethal influenza virus infection. J. Virol. 86, 6792-6803 (2012).

9. Bot, A., Bot, S. \& Bona, C. A. Protective role of gamma interferon during the recall response to influenza virus. J. Virol. 72, 6637-6645 (1998).

10. Graham, M. B. et al. Response to influenza infection in mice with a targeted disruption in the interferon gamma gene. J. Exp. Med. 178, 1725-1732 (1993).

11. Califano, D. et al. IFN-gamma increases susceptibility to influenza A infection through suppression of group II innate lymphoid cells. Mucosal Immunol. 11, 209-219 (2018).

12. Nicol, M. Q. et al. Lack of IFNgamma signaling attenuates spread of influenza $A$ virus in vivo and leads to reduced pathogenesis. Virology 526, 155-164 (2018).

13. McKinstry, K. K. et al. IL-10 deficiency unleashes an influenza-specific Th17 response and enhances survival against high-dose challenge. J. Immunol. 182, 7353-7363 (2009).

14. Eliasson, D. G. et al. M2e-tetramer-specific memory CD4 T cells are broadly protective against influenza infection. Mucosal Immunol. 11, 273-289 (2018).

15. Lord, G. M. et al. T-bet is required for optimal proinflammatory CD4 + T-cell trafficking. Blood 106, 3432-3439 (2005).

16. Strutt, T. M., McKinstry, K. K., Kuang, Y., Bradley, L. M. \& Swain, S. L. Memory CD4 + T-cell-mediated protection depends on secondary effectors that are distinct from and superior to primary effectors. Proc. Natl. Acad. Sci. USA 109, E2551-E2560 (2012).

17. Ghosh, S., Chackerian, A. A., Parker, C. M., Ballantyne, C. M. \& Behar, S. M. The LFA1 adhesion molecule is required for protective immunity during pulmonary Mycobacterium tuberculosis infection. J. Immunol. 176, 4914-4922 (2006).

18. Kohlmeier, J. E. et al. CXCR3 directs antigen-specific effector CD4 $+\mathrm{T}$ cell migration to the lung during parainfluenza virus infection. J. Immunol. 183, 4378-4384 (2009).

19. Mikhak, Z., Strassner, J. P. \& Luster, A. D. Lung dendritic cells imprint T cell lung homing and promote lung immunity through the chemokine receptor CCR4. J. Exp. Med. 210, 1855-1869 (2013).

20. Lazarevic, V., Glimcher, L. H. \& Lord, G. M. T-bet: a bridge between innate and adaptive immunity. Nat. Rev. Immunol. 13, 777-789 (2013).

21. Groom, J. R. et al. CXCR3 chemokine receptor-ligand interactions in the lymph node optimize CD4 + T helper 1 cell differentiation. Immunity 37, 1091-1103 (2012).

22. Wang, X. et al. IL-17A Promotes Pulmonary B-1a Cell Differentiation via Induction of Blimp-1 Expression during Influenza Virus Infection. PLoS Pathog. 12, e1005367 (2016).

23. Crowe, C. R. et al. Critical role of IL-17RA in immunopathology of influenza infection. J. Immunol. 183, 5301-5310 (2009).

24. Marshall, H. D. et al. Differential expression of Ly6C and T-bet distinguish effector and memory Th1 $\mathrm{CD} 4(+)$ cell properties during viral infection. Immunity 35, 633-646 (2011).

25. Hu, Z., Blackman, M. A., Kaye, K. M. \& Usherwood, E. J. Functional heterogeneity in the $\mathrm{CD} 4+\mathrm{T}$ cell response to murine gamma-herpesvirus 68. J. Immunol. 194, 2746-2756 (2015).

26. McKinstry, K. K. et al. Effector CD4 T-cell transition to memory requires late cognate interactions that induce autocrine IL-2. Nat. Commun. 5, 5377 (2014).

27. Dhume, K. \& McKinstry, K. K. Early programming and late-acting checkpoints governing the development of CD4 T-cell memory. Immunology 155, 53-62 (2018).
28. Strutt, T. M. et al. IL-15 supports the generation of protective lung-resident memory CD4 T cells. Mucosal Immunol. 11, 668-680 (2018)

29. Zens, K. D. et al. Reduced generation of lung tissue-resident memory $T$ cells during infancy. J. Exp. Med 214, 2915-2932 (2017).

30. Kamperschroer, C., Dibble, J. P., Meents, D. L., Schwartzberg, P. L. \& Swain, S. L. SAP is required for Th cell function and for immunity to influenza. J. Immunol. 177, 5317-5327 (2006).

31. Strutt, T. M. et al. Direct IL-6 Signals Maximize Protective Secondary CD4 T Cell Responses against Influenza. J. Immunol. 197, 3260-3270 (2016).

32. Strutt, T. M. et al. Memory CD4 + T cells induce innate responses independently of pathogen. Nat. Med. 16, 558-564 (2010). 551p following 564.

33. Rosas, L. E. et al. Cutting edge: STAT1 and T-bet play distinct roles in determining outcome of visceral leishmaniasis caused by Leishmania donovani. J. Immunol. 177, 22-25 (2006).

34. Harms Pritchard, G. et al. Diverse roles for T-bet in the effector responses required for resistance to infection. J. Immunol. 194, 1131-1140 (2015).

35. Sullivan, B. M. et al. Increased susceptibility of mice lacking T-bet to infection with Mycobacterium tuberculosis correlates with increased IL-10 and decreased IFNgamma production. J. Immunol. 175, 4593-4602 (2005).

36. Matsuyama, M. et al. Role of Th1/Th17 balance regulated by T-bet in a mouse model of Mycobacterium avium complex disease. J. Immunol. 192, 1707-1717 (2014).

37. Hultgren, O. H., Verdrengh, M. \& Tarkowski, A. T-box transcription-factor-deficient mice display increased joint pathology and failure of infection control during staphylococcal arthritis. Microbes Infect. 6, 529-535 (2004).

38. Ravindran, R., Foley, J., Stoklasek, T., Glimcher, L. H. \& McSorley, S. J. Expression of T-bet by CD4 T cells is essential for resistance to Salmonella infection. J. Immunol. 175, 4603-4610 (2005).

39. Melillo, A. A., Foreman, O., Bosio, C. M. \& Elkins, K. L. T-bet regulates immunity to Francisella tularensis live vaccine strain infection, particularly in lungs. Infect. Immun. 82, 1477-1490 (2014).

40. Cobb, D. et al. T-bet-dependent regulation of $\mathrm{CD} 8+\mathrm{T}$-cell expansion during experimental Trypanosoma cruzi infection. Immunology 128, 589-599 (2009).

41. Svensson, A., Nordstrom, I., Sun, J. B. \& Eriksson, K. Protective immunity to genital herpes simplex [correction of simpex] virus type 2 infection is mediated by T-bet. J. Immunol. 174, 6266-6273 (2005).

42. Matsui, M., Moriya, O., Yoshimoto, T. \& Akatsuka, T. T-bet is required for protection against vaccinia virus infection. J. Virol. 79, 12798-12806 (2005).

43. Lebrun, A. et al. T-bet Is Required for the Rapid Clearance of Attenuated Rabies Virus from Central Nervous System Tissue. J. Immunol. 195, 4358-4368 (2015).

44. Glanville, N. et al. Thet deficiency causes T helper cell dependent airways eosinophilia and mucus hypersecretion in response to Rhinovirus infection. PLoS Pathog. 12, e1005913 (2016).

45. Yang, Y., Xu, J., Niu, Y., Bromberg, J. S. \& Ding, Y. T-bet and eomesodermin play critical roles in directing $T$ cell differentiation to Th1 versus Th17. J. Immunol. 181, 8700-8710 (2008).

46. Lazarevic, V. et al. T-bet represses $\mathrm{T}(\mathrm{H}) 17$ differentiation by preventing Runx1mediated activation of the gene encoding RORgammat. Nat. Immunol. 12, 96-104 (2011).

47. Intlekofer, A. M. et al. Anomalous type 17 response to viral infection by CD8 + T cells lacking T-bet and eomesodermin. Science 321, 408-411 (2008).

48. Way, S. S. \& Wilson, C. B. Cutting edge: immunity and IFN-gamma production during Listeria monocytogenes infection in the absence of T-bet. J. Immunol. 173, 5918-5922 (2004).

49. Oestreich, K. J. et al. Bcl-6 directly represses the gene program of the glycolysis pathway. Nat. Immunol. 15, 957-964 (2014).

50. van der Windt, G. J. \& Pearce, E. L. Metabolic switching and fuel choice during Tcell differentiation and memory development. Immunol. Rev. 249, 27-42 (2012).

51. Er J. Z., Koean R. A. G., Ding J. L. Loss of T-bet confers survival advantage to influenza-bacterial superinfection. EMBO J. 38, (2018).

52. Fang, D. et al. Transient T-bet expression functionally specifies a distinct $T$ follicular helper subset. J. Exp. Med. 215, 2705-2714 (2018)

53. Knox, J. J., Myles, A. \& Cancro, M. P. T-bet(+) memory B cells: generation, function, and fate. Immunol. Rev. 288, 149-160 (2019).

54. McKinstry, K. K. et al. Rapid default transition of CD4 T cell effectors to functional memory cells. J. Exp. Med. 204, 2199-2211 (2007).

55. Sell, S. et al. Intraepithelial T-cell cytotoxicity, induced bronchus-associated lymphoid tissue, and proliferation of pneumocytes in experimental mouse models of influenza. Viral Immunol. 27, 484-496 (2014). 